\title{
Determination of lifetimes and nonadiabatic correlations from measured dipole polarizabilities
}

\author{
Lorenzo J Curtis \\ Department of Physics and Astronomy, University of Toledo, Toledo, OH 43606, USA
}

Received 27 May 2007, in final form 24 June 2007

Published 25 July 2007

Online at stacks.iop.org/JPhysB/40/3173

\begin{abstract}
In atomic systems for which the total oscillator strength of excitations from the ground state is dominated by the transition to the lowest resonance level, the $f$-sum rule provides a bracketing inequality connecting the lifetime $\tau$ of that level to the dipole polarizability $\alpha_{d}$. This relationship has been used previously to deduce $\alpha_{d}$ from $\tau$. It is shown here that improved spectroscopic accuracies now permit this procedure to be inverted, with $\tau$ deduced from a value for $\alpha_{d}$ obtained spectroscopically using the core polarization model. A similar quantitative relationship exists connecting the nonadiabatic correlation factor $\beta$ to $\tau$, and thus also to $\alpha_{d}$. The method is applied to a recent measurement of $\alpha_{d}$ for $\mathrm{Kr}^{6+}$ to obtain the values $\tau\left(4 \mathrm{~s} 4 \mathrm{p}^{1} \mathrm{P}_{1}\right)=0.096 \pm 0.003 \mathrm{~ns}$ and $\beta\left(\mathrm{Kr}^{6+}\right)=1.71 \pm 0.03 a_{0}^{5}$. It is shown that the use of this method to make precision lifetime determinations for a small number of ions in an isoelectronic sequence permits the exploitation of observed semiempirical regularities to specify the lifetimes of all ions in that sequence.
\end{abstract}

\section{Introduction}

Dipole polarizabilities $\alpha_{d}$ have long been determined from spectroscopic studies of high Rydberg states, interpreted in the context of the core polarization model [1]. Through the study of the energy levels of high $n$ and $\ell$ states, the effective polarizabilities of the core (one stage of ionization higher than the Rydberg ion) can be extracted.

In systems for which the oscillator strength of excitations from the ground state is dominated by the lowest-lying resonance transition, an alternative method can be used to determine $\alpha_{d}$. Using a relationship that connects oscillator strengths and excitation energies with $\alpha_{d}$ and invoking the $f$-sum rule, an inequality can be established. If the dominant resonance transition is unbranched, the value of $\alpha_{d}$ can be tightly bracketed using a precision measurement of the lifetime $\tau$ of that transition (e.g. [2-4]). Similar relationships connect the nonadiabatic correlation factor $\beta$ (a measure of the inability of the core to follow the motion of the outer electron), which allow this quantity to be specified in the same manner. 
This paper demonstrates that improved spectroscopic accuracies now permit the spectroscopic and time-resolved methods to be coupled, and the bracketing procedure inverted. Thus a precision spectroscopic determination of $\alpha_{d}$ using the core polarization model is used to deduce $\tau$, and that result is then used to evaluate $\beta$.

\section{Formulation}

As an illustrative example of this approach, consider the resonance transitions for ions in the $\mathrm{Zn}$ isoelectronic sequence. This system has a $4 \mathrm{~s}^{2}{ }^{1} \mathrm{~S}_{0}$ ground state outside a filled $3 \mathrm{~d}^{10}$ Ni-like core. The oscillator strength for the ground-state excitation series $4 s^{2}{ }^{1} S_{0}-4 s n p{ }^{1} P_{1}^{o}$ is dominated by the strong, low-lying $(\Delta n=0) n=4$ transition. The dipole polarizability of these ions will influence the energy levels of high Rydberg states in atoms and ions of the adjacent $\mathrm{Ga}$ isoelectronic sequence, from which $\alpha_{d}$ can be deduced using the long-range core polarization model [1].

\subsection{Bracketing inequalities}

The value for $\alpha_{d}$ for the ground state of a $\mathrm{Zn}$-like ion can be related to the oscillator strengths $f_{4 \mathrm{~s}, n \mathrm{p}}$ and transition energies $E_{4 \mathrm{~s}, n \mathrm{p}}$ for the $4 \mathrm{~s}^{2}{ }^{1} \mathrm{~S}_{0}-4 \mathrm{~s} n \mathrm{p}{ }^{1} \mathrm{P}_{1}^{\mathrm{o}}$ Rydberg series through the relationship [5]

$$
\alpha_{d}=\sum_{n}^{\prime} \frac{f_{4 \mathrm{~s}, n \mathrm{p}}}{E_{4 \mathrm{~s}, n \mathrm{p}}^{2}},
$$

expressed here in atomic units (energies in Hartrees, lengths in Bohr radii $a_{0}$ ). The prime on the summation indicates that this also includes an integral over continuum states. The $f$-sum rule $[6,7]$ provides the additional relationship

$$
N_{e}=\sum_{n}^{\prime} f_{4 \mathrm{~s}, n \mathrm{p}}
$$

where $N_{e}$ is the number of active electrons.

For ions with $n \mathrm{~s}$ and $n \mathrm{~s}^{2}$ ground states, the oscillator strength of the lowest resonance transition often dominates the sum [3] in equation (2). A possible explanation for this fact involves strong cancellation effects that can occur in the line strength factor for $\Delta n \neq 0$ transitions, but not for the $\Delta n=0$ transition [8]. Since the lowest resonance transition is unbranched, its oscillator strength can be specified from lifetime measurements alone, with no need for branching fraction data. The $f$-sum rule provides a value for the remaining summed oscillator strength, and the second resonance level $4 \mathrm{~s} 5 \mathrm{p}{ }^{1} \mathrm{P}_{1}^{\mathrm{o}}$ provides a lower bound to subsequent excitation energies. This yields a bracketing inequality [9]

$$
\frac{f_{4 \mathrm{~s}, 4 \mathrm{p}}}{E_{4 \mathrm{~s}, 4 \mathrm{p}}^{2}} \leqslant \alpha_{d} \leqslant \frac{f_{4 \mathrm{~s}, 4 \mathrm{p}}}{E_{4 \mathrm{~s}, 4 \mathrm{p}}^{2}}+\frac{N_{e}-f_{4 \mathrm{~s}, 4 \mathrm{p}}}{E_{4 \mathrm{~s}, 5 \mathrm{p}}^{2}} .
$$

This expression has been used to specify values and uncertainty limits for $\alpha_{d}$ from measured lifetime values [2-4]. However, the relationship can also be inverted to yield a bracketing inequality for the oscillator strength

$$
\frac{\alpha_{d} E_{4 \mathrm{~s}, 5 \mathrm{p}}^{2}-N_{e}}{E_{4 \mathrm{~s}, 5 \mathrm{p}}^{2} / E_{4 \mathrm{~s}, 4 \mathrm{p}}^{2}-1} \leqslant f_{4 \mathrm{~s}, 4 \mathrm{p}} \leqslant \alpha_{d} E_{4 \mathrm{~s}, 4 \mathrm{p}}^{2} .
$$

In addition to adiabatic correlations such as $\alpha_{d}$, there are also relationships connecting oscillator strengths to other quantities such as the lowest order nonadiabatic correlation $\beta$ [10]. 
In long-range interactions of a Rydberg ion, this quantity is a measure of the inability of the core to instantaneously follow the motion of the outer electron. It is defined (in au) as

$$
\beta=\frac{1}{2} \sum_{n}^{\prime} \frac{f_{4 \mathrm{~s}, n \mathrm{p}}}{E_{4 \mathrm{~s}, n \mathrm{p}}^{3}} .
$$

By the same arguments that were presented above for $\alpha_{d}$, the quantity $\beta$ has the bracketing inequality

$$
\frac{f_{4 \mathrm{~s}, 4 \mathrm{p}}}{E_{4 \mathrm{~s}, 4 \mathrm{p}}^{3}} \leqslant 2 \beta \leqslant \frac{f_{4 \mathrm{~s}, 4 \mathrm{p}}}{E_{4 \mathrm{~s}, 4 \mathrm{p}}^{3}}+\frac{N_{e}-f_{4 \mathrm{~s}, 4 \mathrm{p}}}{E_{4 \mathrm{~s}, 5 \mathrm{p}}^{3}} .
$$

After the measured value for $\alpha_{d}$ has been used to deduce $f_{4 \mathrm{~s} 4 \mathrm{p}}$, the process can be reversed to prescribe the value for $\beta$.

\section{Quantitative application}

As a numerical application of this approach, consider the recent measurement by Lundeen and Fehrenbach [11] of a precise (1.5\%) value of $\alpha_{d}$ for $\mathrm{Zn}$-like $\mathrm{Kr}^{6+}$. This was done using high-resolution spectroscopy of high Rydberg levels of $\mathrm{Kr}^{5+}$, interpreted in the context of the long-range core polarization model [1].

The lifetime of the $4 \mathrm{~s} 4 \mathrm{p}^{1} \mathrm{P}_{1}^{\mathrm{o}}$ level is particularly interesting because its measurement by decay curve analysis is complicated by heavy cascade repopulation. Early measurements using multiexponential curve fitting yielded a value $0.19 \pm 0.02$ ns $[12,13]$. A later measurement that used the ANDC method [14] to make a correlated analysis of the primary and cascade decay curves yielded a value $0.101 \pm 0.010 \mathrm{~ns}$ [15]. However, a subsequent study [16] suggested that one of the lines attributed to a relevant cascade channel may have been misidentified. While the inherent stimulus-response correlation between the cascade and primary decay curves makes misidentification in a successful ANDC analysis extremely unlikely, an independent determination of this lifetime could also confirm the identification. Theoretical calculations have yielded values $0.0991 \mathrm{~ns}$ [17] and $0.0953 \mathrm{~ns}$ [18].

The expressions developed in the previous section can be applied to this new measurement of $\alpha_{d}$ for $\mathrm{Kr}^{6+}$. Values for the $n=4$ and 5 energies are available $\left(E_{4 \mathrm{~s}, 4 \mathrm{p}}=0.778382 \mathrm{au}\right.$ [19] and $E_{4 \mathrm{~s}, 5 \mathrm{p}}=2.267961 \mathrm{au}$ [16]), and Lundeen and Fehrenbach [11] provide the value $\alpha_{d}=2.69 \pm 0.04 a_{0}^{3}$.

\subsection{Evaluation of the $f$-sum rule}

The only remaining unknown is $N_{e}$, which can be expected to be close to two, the number of out-of-shell $4 \mathrm{~s}^{2}$ electrons in the ground state. There could also be small additional contributions from the closed-shell $3 \mathrm{~d}^{10} \mathrm{Ni}$-like $\mathrm{Kr}^{8+}$ inner core. However, the $\mathrm{Kr}^{8+}$ ion is relatively rigid, with a calculated polarizability $\alpha_{d}=0.2021 a_{0}^{3}$ [20]. To account for the possibility of an inner core contribution to the oscillator strength of $\mathrm{Kr}^{6+}$, denote

$$
N_{e}=2+\Delta N_{e}
$$

where $\Delta N_{e}$ is a small fraction. For example, in studies of the $f$-value sums for $\mathrm{Mg}^{+}$and $\mathrm{Ca}^{+}$ [3], values $\Delta N_{e} \approx 0.1$ were obtained.

Another consideration that could affect the validity of the $f$-sum rule is the degree to which intermediate coupling produces singlet-triplet mixing between the $4 \mathrm{~s} 4 \mathrm{p}^{1} \mathrm{P}_{1}^{\mathrm{o}}$ and ${ }^{3} \mathrm{P}_{1}^{\mathrm{o}}$ levels. This can be investigated by extracting the mixing amplitudes from the measured energy spacings [21] among the $4 \mathrm{~s} 4 \mathrm{p}^{3} \mathrm{P}_{2}^{\mathrm{o}},{ }^{3} \mathrm{P}_{1}^{\mathrm{o}},{ }^{1} \mathrm{P}_{0}^{\mathrm{o}}$ levels (0.534853 au, $\left.0.547192 \mathrm{au}, 0.576662 \mathrm{au}\right)$ 
and the $4 s 4 p^{1} \mathrm{P}_{1}^{o}$ level (see above). Since there are three intervals and only two amplitudes, the system is overdetermined. The two normalized amplitudes can be expressed as a single mixing angle $\theta$. A solution involving only two of the splittings (taking the average energy of the $J=1$ levels) is given by

$$
\cot (2 \theta)= \pm \frac{1}{\sqrt{2}}\left[\frac{3\left({ }^{3} \mathrm{P}_{1}^{\mathrm{o}}+{ }^{1} \mathrm{P}_{1}^{\mathrm{o}}-2^{3} \mathrm{P}_{0}^{\mathrm{o}}\right)}{\left({ }^{3} \mathrm{P}_{2}^{\mathrm{o}}-{ }^{3} \mathrm{P}_{0}^{\mathrm{o}}\right)}-1\right]
$$

(here the energy levels are designated by their spectroscopic symbols). This yields a value $\tan \theta=0.0857$ which, combined with the ratio of the ${ }^{3} \mathrm{P}_{1}^{\mathrm{o}}$ and ${ }^{1} \mathrm{P}_{1}^{\mathrm{o}}$ excitation energies, indicates that singlet-triplet mixing decreases the oscillator strength of the resonance line by only $0.52 \%$. Checking the overdetermination, the mixing amplitudes obtained from equation (8) reproduced the ${ }^{1} \mathrm{P}_{1}^{\mathrm{o}}-{ }^{3} \mathrm{P}_{1}^{\mathrm{o}}$ splitting to within $0.082 \%$, consistent with the assumption of a pure single configuration.

\subsection{Determination of the oscillator strength and lifetime}

Inserting equation (7) and the values for $E_{4 \mathrm{~s}, 4 \mathrm{p}}, E_{4 \mathrm{~s}, 5 \mathrm{p}}$ and $\alpha_{d}$ into equation (4),

$$
1.5804-0.1335 \Delta N_{e} \leqslant f_{4 \mathrm{~s}, 4 \mathrm{p}} \leqslant 1.6298 \text {. }
$$

As can be seen from equations (3) and (6), $N_{e}$ affects only the upper bound on the bracketing of $\alpha_{d}$ and $\beta$. Since equation (4) is an inversion of equation (3), only the lower bound of $f_{4 \mathrm{~s}, 4 \mathrm{p}}$ is affected by $N_{e}$. Thus there is a slight downward shift with increasing $\Delta N_{e}$ for the lower bound of $f_{4 \mathrm{~s}, 4 \mathrm{p}}$. For $\Delta N_{e}=0.1$, the lower bound shifts from 1.5804 to 1.5670 , a $0.85 \%$ reduction. If we accept this as a reasonable value for $\Delta N_{e}$, the inequality becomes

$$
1.5670 \leqslant f_{4 \mathrm{~s}, 4 \mathrm{p}} \leqslant 1.6298,
$$

which has a centroid $f_{4 \mathrm{~s}, 4 \mathrm{p}}=1.598$ and a spread of \pm 0.031 , or $2 \%$. Since this determination involves inequalities rather than equalities, this value for $f_{4 \mathrm{~s}, 4 \mathrm{p}}$ was inserted into equation (3) to test reciprocity. This recovered a value for $\alpha_{d}$ of $2.687 a_{0}^{3}$, consistent within the tight bracketing of the inequalities. Tests using $\Delta N_{e}=0$ and 0.2 were also made, which shifted the value of $f_{4 \mathrm{~s}, 4 \mathrm{p}}$ by only \pm 0.007 .

If the bracketing half-width is combined in quadrature with the $1.5 \%$ uncertainty in the measurement of $\alpha_{d}$, the value deduced is

$$
f_{4 \mathrm{~s}, 4 \mathrm{p}}=1.60 \pm 0.04 \text {. }
$$

The fact that 1.6 units of the oscillator strength reside in $f_{4 \mathrm{~s}, 4 \mathrm{p}}$ means that there are only $\approx 0.5$ units of oscillator strength spread over all of the other bound and continuum states. A lifetime measurement [16] for the $4 \mathrm{~s} 5 \mathrm{p}^{1} \mathrm{P}_{1}^{\mathrm{o}}$ level yields an upper limit (assuming $100 \%$ branching to ground) $f_{4 \mathrm{~s}, 5 \mathrm{p}} \leqslant 0.063$, illustrating the dominance of $f_{4 \mathrm{~s}, 4 \mathrm{p}}$ in the sum. Moreover, the low-lying intrashell nature of the $n=4$ transition leads to a large ratio $E_{4 \mathrm{~s}, 5 \mathrm{p}}^{2} / E_{4 \mathrm{~s}, 4 \mathrm{p}}^{2} \approx 8.5$, further decreasing the contribution of higher-lying levels to the inequality. For these reasons, the bracketing inequality is very tight, and relatively insensitive to $\Delta N_{e}$.

For an unbranched transition, the relationship [22] between the lifetime and the oscillator strength is (energy in au)

$$
\frac{g_{4 \mathrm{p}}}{\tau_{4 \mathrm{p}}}=\frac{2 c \alpha^{4}}{a_{0}} E_{4 \mathrm{~s}, 4 \mathrm{p}}^{2} g_{4 \mathrm{~s}} f_{4 \mathrm{~s}, 4 \mathrm{p}}
$$

where $g_{k}$ denotes the degeneracies of the levels and $\alpha$ is the fine structure constant. Making this conversion, the result in equation (11) corresponds to a lifetime

$$
\tau_{4 \mathrm{p}}=0.096 \pm 0.003 \mathrm{~ns} .
$$


This confirms the ANDC measurement $0.101 \pm 0.010 \mathrm{~ns}$ [15] with a significant improvement in accuracy, and also corroborates the correctness of the identification of the cascade in that ANDC analysis. The value is also in good agreement with theoretical estimates $[17,18]$.

\subsection{Determination of the nonadiabatic correlation}

Having established the value of $f_{4 \mathrm{~s}, 4 \mathrm{p}}$, this can be used in equation (6) to bracket the value of $\beta$. This yields values

$$
1.6947 \leqslant \beta \leqslant 1.7119+0.0428 \Delta N_{e} .
$$

Because equation (6) involves the cube of the excitation energies and $E_{4 \mathrm{~s}, 5 \mathrm{p}}^{3} / E_{4 \mathrm{~s}, 4 \mathrm{p}}^{3} \approx 25$, the effect of $\Delta N_{e}$ on the bracketing is even less in the case of $\beta$. Again using the estimate $\Delta N_{e}=0.1$

$$
1.6947 \leqslant \beta \leqslant 1.7162 \text {. }
$$

Taking the central value and combining the bracketing width in quadrature with the uncertainty in the measurement of $\alpha_{d}$, the value obtained is

$$
\beta=1.71 \pm 0.03 a_{0}^{5} \text {. }
$$

Theoretical values for this quantity are not available, but such calculations could provide a useful test of the method.

\section{Application to isoelectronic sequences}

The quantum-mechanical aspects of both the emission lifetime $\tau_{4 \mathrm{p}}$ and the absorption oscillator strength $f_{4 \mathrm{~s}, 4 \mathrm{p}}$ are derived from the dipole transition element as expressed in the line strength factor $S_{4 \mathrm{~s}, 4 \mathrm{p}}$ [22]:

$$
S_{4 \mathrm{~s}, 4 \mathrm{p}}=S_{4 \mathrm{p}, 4 \mathrm{~s}}=\left|\left\langle\Psi_{4 \mathrm{~s}}|\mathbf{r}| \Psi_{4 \mathrm{p}}\right\rangle\right|^{2} .
$$

While this quantity is theoretically defined, it can alternatively be treated as an empiricallydetermined experimental parameter. It can be deduced from the oscillator strength using $[22]$

$$
g_{4 \mathrm{~s}} f_{4 \mathrm{~s}, 4 \mathrm{p}}=\frac{2}{3} E_{4 \mathrm{~s}, 4 \mathrm{p}} S_{4 \mathrm{~s}, 4 \mathrm{p}}
$$

(energy in $\mathrm{au}$ ), where $f_{4 \mathrm{~s}, 4 \mathrm{p}}$ is determined either from the measured value of $\tau_{4 \mathrm{p}}$ using equation (12), or from the measured value of $\alpha_{d}$ using equation (4).

There is much empirical evidence [23-27] indicating that ions with one or two out-ofshell electrons exhibit a nearly linear isoelectronic variation when the line strength factor is scaled quadratically with the nuclear charge $Z$ and plotted against a suitably chosen reciprocal screened charge. Thus

$$
Z^{2} S=S_{H}+B /(Z-C)
$$

where the trend approaches the hydrogenic limit $S_{H}$ for large $Z$ [23]. An example is shown in figure 1. The solid diamond represents the hydrogenic value $S_{H}=1080$.

In the case of two-valence-electron systems, it is also possible to take into account the effects of intermediate coupling [24] using the singlet-triplet mixing angle obtained from equation (8). First, line strength factors $S$ (Res) and $S$ (Int) are deduced from measured lifetimes for the resonance and intercombination transitions. These are then corrected for the effects of intermediate coupling to obtain effective line strengths $S_{r}$ (Res) and $S_{r}$ (Int) using the relationships

$$
S_{r}(\operatorname{Res}) \equiv S(\operatorname{Res}) / \cos ^{2} \theta \quad S_{r}(\text { Int }) \equiv S(\operatorname{Int}) / \sin ^{2} \theta .
$$




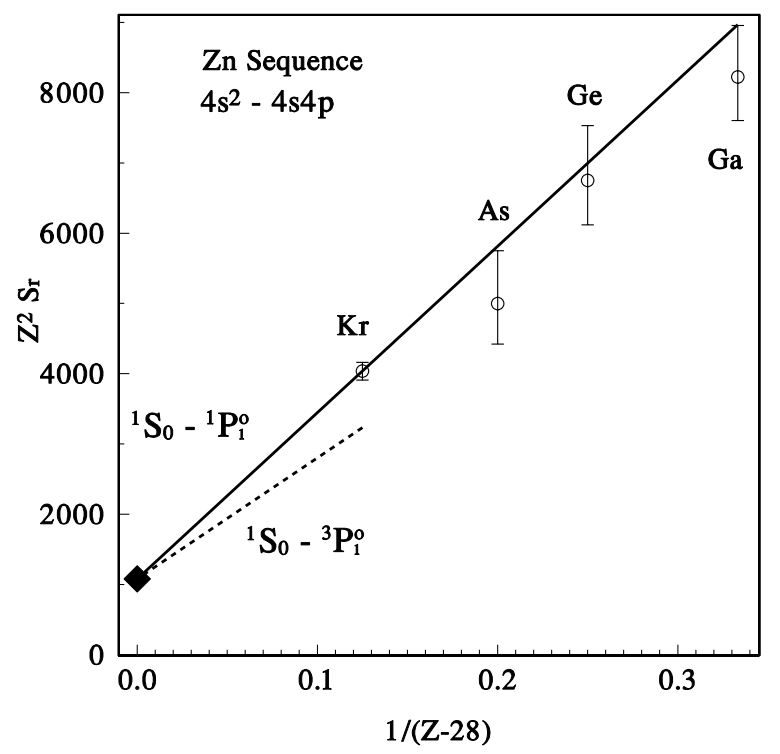

Figure 1. Isoelectric interpolation of the reduced line strengths for the $4 s^{2}-4 s 4 p$ transitions in the Zn sequence.

This approach was applied to the $\mathrm{Zn}$ sequence earlier [25]. Based on lifetime measurements (generally of $\approx 10 \%$ precision) of the resonance transitions in Ga II, Ge III, As IV and $\mathrm{Kr}$ VII, and of the intercombination transitions in Kr VII, Nb XII, Mo XIII and Ag XVIII, empirical lifetimes were predicted for all charged ions from gallium to uranium. The precise determination of the $\mathrm{Kr}$ VII lifetime reported here provides a means to substantially improve the determination of the singlet lifetimes in this sequence, as is evident from figure 1.

In the past this approach has been hindered by the lack of high precision measurements for multiply-charged ions. Measurements at the $1 \%$ level are largely limited to neutral and singly ionized atoms [28], where methods such as laser selective excitation of a static sample can be employed. However, the regularity exhibited by isoelectronic data expositions occurs for multiply-ionized systems, where the interactions are predominantly central. Most measurements in multiply-charged ions are made through non-selective excitation of a fast ion beam. The uncertainties in this type of measurement are typically 5-10\%, often increasing with degree of ionicity. Thus the use of the method described here to obtain a $3 \%$ measurement in a six-times ionized atom represents a significant advance in the predictive accuracy of this method.

It is clear from figure 1 that the Kr VII point, together with the hydrogenic asymptote at high $Z$, establishes the fit to unprecedented accuracy. If measurements of modest accuracy are sufficient to establish a screening constant $C$ that produces linearity in this plotting exposition, then a single measurement of high accuracy can be connected with the hydrogenic asymptote to specify all members of the sequence. If a second ion in this sequence could be measured to this precision, it would provide a means of confirming the linearity and further sharpening the predictions.

Similar predictive semiempirical parametrizations of measured data have been carried out for the Mg [24], Cd [26] and $\mathrm{Hg}$ [27] sequences. These studies could be greatly improved by the addition of lifetime values deduced from precision spectroscopic determinations of $\alpha_{d}$ using the methods described here. When a comprehensive isoelectronic parametrization has 
been made for $\tau$, the results can be utilized to provide a similar isoelectronic data base for $\alpha_{d}$ and $\beta$.

\section{Accuracy of the method}

As has been described above, the accuracy of this method involves the uncertainties in the measured quantities, the magnitude of and the uncertainties in the width of the bracketing inequality, and the validity of the single configuration approximation. Uncertainties in the energy levels are usually well below the $1 \%$ level and do not contribute to the uncertainty. The single configuration approximation can be tested using the overdetermination of the level separations.

The width of the bracketing inequality (and hence the influence of its uncertainty) will be small if: $f_{4 \mathrm{~s}, 4 \mathrm{p}}$ is large; $E_{4 \mathrm{~s}, 4 \mathrm{p}}$ is small; and $E_{4 \mathrm{~s}, 5 \mathrm{p}}$ is close to the ionization limit. For ions of the $\mathrm{Mg}, \mathrm{Zn}, \mathrm{Cd}$ and $\mathrm{Hg}$ sequences these criteria are generally satisfied, and the propagation of uncertainties within the method should be comparable to or smaller than the experimental uncertainties in a 1-2\% spectroscopic determination of $\alpha_{d}$.

Additional tests of the method could be made if precision time-resolved measurements of $\tau$ and precision spectroscopic measurements of $\alpha_{d}$ could both be performed for the same ion, allowing a cross-check of consistency and reciprocity. The accuracy of the method could also be probed by theoretical investigations of the magnitude of inner-shell contributions to ground-state oscillator strengths.

\section{Conclusion}

For ionic systems for which the ground-state oscillator strength is concentrated in the lowestlying resonance transition, this method provides a means to interconnect $\alpha_{d}, \beta$ and $\tau$. If, as in this case, a precision measurement of $\alpha_{d}$ is available, $\tau$ and $\beta$ can be deduced. Alternatively, if a precision measurement of $\tau$ is available, $\alpha_{d}$ and $\beta$ can be specified. Moreover, screening parametrizations of reduced-line-strength data have been shown to permit accurate isoelectronic interpolation and smoothing for entire isoelectronic sequences, based on a small number of precisely measured lifetimes. This method could permit similar isoelectronic specification $\alpha_{d}$ and $\beta$. These quantities could also be efficiently deduced from $a b$ initio theoretical calculations of the intrashell oscillator strength.

It is hoped that the opportunity to exploit these interconnections will stimulate additional precision spectroscopic studies of this type in similar systems.

\section{References}

[1] Edlén B 1964 Atomic spectra Handb. der Physik XXVII ed S Flügge (Berlin: Springer) section 20, pp 80-220

[2] Curtis L J and Theodosiou C E 1995 J. Opt. Soc. Am. B 12 175-7

[3] Theodosiou C E, Curtis L J and Nicolaides C A 1995 Phys. Rev. A 52 3677-80

[4] Henderson M, Curtis L J, Matulioniene R, Ellis D G and Theodosiou C E 1997 Phys. Rev. A 56 1872-8

[5] Dalgarno A and Kingston A E 1959 Proc. Phys. Soc. 74 455-64

[6] Kuhn W 1925 Z. Phys. 33 408-12

[7] Reiche F and Thomas W 1925 Z. Phys. 34 510-25

[8] Curtis L J 1991 Can. J. Phys. $69668-70$

[9] Miller T M and Bederson B 1977 Adv. At. Mol. Phys. 13 1-55

[10] Kleinman C J, Hahn Y and Spruch L 1968 Phys. Rev. 165 53-62

[11] Lundeen S R and Fahrenbach C W 2007 Phys. Rev. A 75 032523:1-7

[12] Druetta M and Buchet J P 1976 J. Opt. Soc. Am. B 66 433-6 
[13] Knystautas E J and Drouin R 1977 J. Quant. Spectrosc. Radiat. Transfer 17 551-3

[14] Curtis L J, Berry H G and Bromander J 1971 Phys. Lett. 34A 169-70

[15] Pinnington E H, Ansbacher W and Kernahan J A 1984 J. Opt. Soc. Am. B 1 30-3

[16] Pinnington E H, Tauheed A, Ansbacher W and Kernahan J A 1991 J. Opt. Soc. Am. B 8 193-7

[17] Liu Y, Hutton R, Zou Y, Andersson M and Brage T 2006 J. Phys. B: At. Mol. Opt. Phys. 39 3147-58

[18] Chou H-S, Chi H-C and Huang K-N 1994 Phys. Rev. A 49 2394-8

[19] NIST Physical Reference Data http://physics.nist.gov/PhysRefData/contents.html

[20] Johnson W R, Kolb D and Huang K-N 1983 At. Data Nucl. Data Tables 28 333-40

[21] Curtis L J 1989 Phys. Rev. A 40 6958-68

[22] Curtis L J 2003 Atomic Structure and Lifetimes: A Conceptual Approach (Cambridge: Cambridge University Press)

[23] Curtis L J, Ellis D G and Martinson I 1995 Phys. Rev. A 51 251-6

[24] Curtis L J 1991 Phys. Scr. 43 137-43

[25] Curtis L 1992 J. Opt. Soc. Am. 9 5-9

[26] Curtis L J, Matulioniene R, Ellis D G and Froese Fischer C 2000 Phys. Rev. A 62 052513:1-7

[27] Curtis L J, Irving R E, Henderson M, Matulioniene R, Froese Fischer C and Pinnington E 2001 Phys. Rev. A 63 042502:1-7

[28] Curtis L J 2006 Precision oscillator strength and lifetime measurements Handbook of Atomic, Molecular and Optical Physics ed G W F Drake (New York: Springer) chapter 17, pp 261-8 